EGU21-14539

EGU General Assembly 2021

(c) Author(s) 2021. This work is distributed under

the Creative Commons Attribution 4.0 License.

\title{
Approaches to improve the robustness of the Comparison Model Method for the inverse problem of groundwater hydrology
}

\begin{abstract}
Alessandro Comunian and Mauro Giudici
Università degli Studi di Milano, Department of Earth Sciences, Milan, Italy (alessandro.comunian@unimi.it)

Indirect inversion approaches are widely used in Geosciences, and in particular also for the identification of the hydraulic properties of aquifers. Nevertheless, their application requires a substantial number of model evaluation (forward problem) runs, a task that for complex problems can be computationally intensive. Reducing this computational burden is an active research topic, and many solutions, including the use of hybrid optimization methods, the use of physical proxies or again machine-learning tools allow to avoid considering the full physics of the problem when running a numerical implementation of the forward problem.
\end{abstract}

Direct inversion approaches represent computationally frugal alternatives to indirect approaches, because in general they require a smaller number of runs of the forward problem. The classical drawbacks of these methods can be alleviated by some implementation approaches and in particular by using multiple sets of data, when available.

This work is an effort to improve the robustness of the Comparison Model Method (CMM), a direct inversion approach aimed at the identification of the hydraulic transmissivity of a confined aquifer. The robustness of the CMM is here ameliorated by (i) improving the parameterization required to handle small hydraulic gradients; (ii) investigating the role of different criteria aimed at merging multiple data-sets corresponding to different flow conditions.

On a synthetic case study, it is demonstrated that correcting a small percentage of the small hydraulic gradients (about 10\%) allows to obtain reliable results, and that a criteria based on the geometric mean is adequate to merge the results coming from multiple data-sets. In addition, the use of multiple-data sets allows to noticeably improve the robustness of the CMM when the input data are affected by noise.

All the tests are performed by using open source and widely used tools like the USGS Modflow6 and its Python interface flopy to foster the application of the CMM. The scripts and corresponding package, named $\mathrm{cm} m p y$, is available on the Python Package Index (PyPI) and on bitbucket at the following address: https://bitbucket.org/alecomunian/cmmpy. 